\title{
La Sociedad Anónima Europea (SE). ¿Una oportunidad perdida para la democracia industrial en europa?
}

\author{
Sergio GONZÁLEZ BEGEGA \\ Universidad de Oviedo. Departamento de Sociología \\ gonzalezsergio@uniovi.es \\ Holm-Detlev KÖHLER \\ Universidad de Oviedo. Departamento de Sociología \\ hkohler@uniovi.es
}

Recibido: 08-10-2013

Aceptado: 20-01-2014

\section{RESUMEN}

La adopción en 2001 del Estatuto de Sociedad Anónima Europea supuso la culminación de un objetivo largamente perseguido por las instituciones comunitarias. Además de dotar a los socios de la Unión Europea de un vehículo jurídico único para la constitución de sociedades, el Estatuto de Sociedad Anónima Europea se vio suplementado por una Directiva de representación laboral que fue recibida como una pieza de gran potencial para el desarrollo de las relaciones laborales europeas. Algo más de una década más tarde, las expectativas generadas en torno a este reglamento no se han visto satisfechas. La regulación no ha tenido el efecto de transformación sobre las prácticas de representación laboral en la toma de decisiones corporativas transnacionales que algunos le atribuyeron en el momento de su aprobación. El artículo examina críticamente los motivos por los cuales el Estatuto de Sociedad Europea no ha cumplido su potencial transformador.

Palabras clave: relaciones laborales europeas, empresa transnacional, toma de decisiones corporativas, participación laboral, democracia industrial.

\section{The European Society (SE). A missed opportunity for industrial democracy in europe?}

\begin{abstract}
The adoption of the European Society Statute in 2001 fulfilled a long pursued goal of the European Union institutions. It provided companies with a single legal tool to register in all member states. Besides, the European Society Statute was supplemented with a Directive on workers' involvement which was seen as legal piece with many possibilities for the future development of European industrial relations. Those expectations have not been met a decade and almost a half after. The regulation has not achieved the transformational effects on the practice of employee representation at corporate decision-making that some experts and practitioners foresaw by the time it was passed. The article evaluates the reasons for this failure. The track of the European Society Statute is reconstructed in order to explain why its alleged capacity for transformation has not been shown.
\end{abstract}

Keywords: European industrial relations, transnational company, corporate decision-making, workers' involvement, industrial democracy. 


\section{REFERENCIA NORMALIZADA}

González Begega, S., Köhler, H.-D. (2015). “La Sociedad Anónima Europea (SE). ¿Una oportunidad perdida para la democracia industrial en Europa?”. Cuadernos de Relaciones Laborales, Vol. 33, núm. 1, p. 65-91.

SUMARIO: Introducción. Expectativas y juicios sobre la Sociedad Europea. 1. En las raíces del proyecto comunitario. La génesis política del Estatuto de Sociedad Europea. 2. La Sociedad Europea y su Directiva de implicación laboral. Principales aspectos normativos. 3. La Sociedad Europea. Variedades operativas y despliegue práctico. 4. Conclusiones. ¿Motivos para la frustración? El largo impasse de las relaciones laborales europeas. 5. Bibliografía.

\section{Introducción. Expectativas y juicios sobre la Sociedad Europea}

La adopción en 2001, por parte del Consejo de Europa, del Estatuto de la Sociedad Anónima Europea supuso la culminación de un esfuerzo político por parte de las instituciones comunitarias que hunde sus raíces en la década de los años cincuenta ${ }^{1}$. El Estatuto de Sociedad Europea proporciona a las empresas la posibilidad de registrase bajo una misma forma societaria en todos los Estados miembros de la Unión. Desde la perspectiva de los intereses laborales, el Estatuto de Sociedad Anónima Europea se vio acompañado de una Directiva de representación de trabajadores a escala de consejo de administración de la empresa ${ }^{2}$. El esfuerzo regulador fue completado dos años más tarde, en 2003, con la aprobación del Estatuto de Sociedad Cooperativa Europea, que también fue acompañado de una Directiva de participación laboral ${ }^{3}$.

Una década después de la aprobación de ambas piezas legales, las expectativas generadas sobre su capacidad de transformación de la realidad societaria y laboral europea no se han visto satisfechas. A comienzos de 2013, el número de empresas voluntariamente constituidas como Sociedades Anónimas Europeas o como Sociedades Cooperativas Europeas se sitúa en torno a las 1600. La cifra, aparentemente significativa, puede llevar a equívocos. La existencia de importantes vacíos normativos ha restado interés a la Sociedad Europea como fórmula de constitución societaria y, sobre todo, ha impedido que la regulación laboral que la

\footnotetext{
${ }^{1}$ Reglamento del Consejo de Europa (CE) 2157/2001, de 8 de octubre de 2001, por el que se aprueba el Estatuto de la Sociedad Anónima Europea (SE). DO L 294 de 10/11/2001, p. 1-21.

${ }^{2}$ Directiva del Consejo de Europa 2001/86/CE, de 8 de octubre de 2001, por la que se completa el Estatuto de la Sociedad Anónima Europea en lo que respecta a la implicación de los trabajadores. DO L 294 de 10/11/2001, p. 22-32.

${ }^{3}$ Respectivamente, Reglamento del Consejo de Europa (CE) 1435/2003, de 22 de julio de 2003, relativo al Estatuto de la Sociedad Cooperativa Europea. DO L 207 de 18/08/2003, p. 1-39. Rectificación DO L 49 de 17/02/2007, p. 35; y Directiva del Consejo de Europa 2003/72/CE, de 22 de julio de 2003, por la que se completa el Estatuto de la Sociedad Cooperativa Europea en lo que respecta a la implicación de los trabajadores. DO L 207 de 18/08/2003, p. 25-36.
} 
acompaña haya tenido el efecto transformador sobre las prácticas de representación de trabajadores en la toma de decisiones corporativas que le fueron atribuidas en el momento de su adopción. Las Directivas de participación de trabajadores asociadas a los Estatutos de Sociedad Anónima Europea y Sociedad Cooperativa Europea (en adelante referidos como Sociedad Europea, SE) proporcionaban, a juicio de expertos y legisladores, un punto de partida normativo para homogeneizar al alza los derechos de participación de los trabajadores en la toma de decisiones corporativas, al recoger la reserva de asientos a escala de consejo de administración para la representación del interés laboral.

Para algunos expertos decididamente entusiastas, la SE iba a subsanar las incoherencias de la normativa sobre comités de empresa europeos, que hasta entonces había constituido la punta de lanza de las relaciones laborales europeas a escala de empresa (Carley y Bockler, 1998) ${ }^{4}$. Las Directivas de participación laboral de la SE, al extender los derechos de información y consulta de los empleados más allá de la información y consulta y reconocer la incorporación estatutaria de los intereses de los trabajadores al máximo órgano de toma de decisiones de la empresa, se inspiraban en los sistemas de participación de empleados más avanzados en Europa y expedían el camino hacia una democracia industrial a escala europea (Köstler y Buggel, 2003). En cuanto a las ventajas de la forma societaria recogida por la SE, Nöelle Lenoir (2008: 20) argumentaba que, "a pesar de algunas imperfecciones perfectamente subsanables (...) ofrecía evidente interés para las auténticas empresas europeas o para las subsidiarias europeas de empresas norteamericanas o japonesas”. Lenoir (2007) señalaba además que la SE constituía un soporte normativo vigoroso para avanzar en la movilidad empresarial y en la construcción de una identidad común de la empresa europea.

El análisis de la evidencia disponible sobre la SE muestra que estas expectativas optimistas han resultado inadecuadamente satisfechas. Los resultados de la SE, más allá de su carácter de portaestandarte del derecho societario europeo (Wenz, 2004), deben ser interpretados con mayor cautela, tanto en su impacto sobre el derecho de sociedades (Linmondin, 2003) como, sobre todo, en un sentido laboral. El mapa de uso de la SE ofrece fuertes discontinuidades según país, por la orientación específica del derecho de sociedades dentro de cada Estado miembro y por la diversidad de tradiciones de relaciones laborales (Stollt y Kluge, 2011a). La homogeneización prometida por la SE ha debido hacer frente a importantes

${ }^{4}$ Directiva del Consejo de Europa 94/45/CE, de 22 de septiembre de 1994, sobre la constitución de un comité de empresa europeo o de un procedimiento de información y consulta a los trabajadores en las empresas y grupos de empresas de dimensión comunitaria. DO L 254 de 30/09/1994, p. 64-72. Revisada por la Directiva 2009/38/CE del Parlamento Europeo y del Consejo de Europa, de 6 de mayo de 2009, sobre la constitución de un comité de empresa europeo o de un procedimiento de información y consulta a los trabajadores en las empresas y grupos de empresas de dimensión comunitaria. DO L 122 de 16/05/2009, p. 28-44. 
dificultades de implementado nacional, que han lastrado su capacidad para facilitar la restructuración corporativa transnacional (Esteban Velasco, 2003) y han afectado negativamente a la efectividad de los ambiciosos derechos de representación laboral que la suplementan. Buena prueba del reconocimiento por parte del legislador de esta segunda insatisfacción fue la apertura en junio de 2011 de una ronda de consultas entre la Comisión Europea y los agentes sociales europeos para una eventual revisión de las Directivas sobre la implicación de trabajadores en la SE. La iniciativa, como se detalla en el apartado correspondiente del artículo, se encuentra actualmente en un punto muerto.

La evaluación del impacto de la SE sobre las relaciones laborales europeas revela un cuadro ambiguo. Por un lado, el impacto general resulta limitado dado el bajo número de Sociedades Europeas constituidas en la mayor parte de países (...). Por otro lado, la SE ha ampliado significativamente los derechos de los trabajadores y ha contribuido a la extensión de una dimensión europea de la implicación de los empleados (Stollt y Kluge, 2011b: 188).

El artículo examina la incidencia de la normativa sobre la SE sobre los derechos de representación de los trabajadores en la empresa transnacional y, particularmente, la participación laboral en la toma de decisiones corporativas.

La estructura del mismo será la siguiente. En primer lugar, se reconstruirá el proceso político conducente la adopción de la regulación sobre la SE en 2001, tras una negociación que se prolonga de manera discontinua durante cuatro décadas, entrelazándose con uno de los objetivos fundacionales de la Unión Europea: la unidad de mercado. A continuación, se analizarán las características de la normativa de participación laboral que acompaña a la SE, examinando asimismo la, por ahora, abortada iniciativa de recalibrado de las Directivas sobre implicación de trabajadores en la SE. En este mismo apartado, se examinará brevemente el proceso de transposición de la normativa sobre la SE al marco jurídico español. En la tercera sección, se acotarán las dimensiones cuantitativas del proceso de constitución de Sociedades Europeas, abordando los modelos operativos de SE y tratando de explicar la desigual atención prestada al proceso de la SE en los países cubiertos por la normativa. La marginalidad de la SE en el Sur de Europa contrasta con el significativo número de SE constituidas en Alemania o la República Checa. A partir de la evidencia disponible, se explicarán las causas del desequilibrado impacto geográfico de la SE. A modo de conclusión, se retomarán las cuestiones de juicio planteadas en esta introducción en torno al significado de la SE para la representación de intereses laborales en la toma de decisiones corporativas en la Unión Europea. Asimismo, se efectuará una consideración más general acerca del largo momento de impasse que actualmente atraviesan las relaciones laborales europeas. 


\section{En las raíces del proyecto comunitario. La génesis política del Estatuto de Sociedad Europea}

La negociación política del reglamento de la SE abarca más de cuarenta años de la historia del proyecto europeo. A lo largo de este prolongado periodo, las frustraciones acumuladas y la dificultad de concretar los objetivos políticos se manifiestan especialmente en los aspectos societarios y laborales insatisfactorios de las piezas legales aprobadas finalmente a comienzos de la década de los dos mil. La tensión entre autoridades comunitarias, poderes públicos nacionales y actores privados explica el pragmatismo de la regulación sobre la SE adoptada finalmente $\mathrm{y}$, también, en buena medida, su ambiguo impacto.

El rastro de la SE se entrecruza con la tortuosa historia de la construcción política europea y las dificultades de homogeneización normativa. La SE representa, en primer lugar, un proyecto de regulación interior estrechamente asociado al objetivo de construcción del mercado común europeo. Desde los primeros debates sobre la SE, que se producen en la década de los sesenta, lo que se encuentra en juego es el desarrollo de un instrumento societario que dote de flexibilidad a las empresas que operan a escala transfronteriza en Europa. En términos jerárquicos, ese es el primer objetivo de la SE, aunque sus efectos sobre el ámbito de la regulación laboral no sean desdeñables (Stollt y Kluge, 2011b).

En el debate sobre la SE también está presente el germen de una idea de relaciones laborales europeas, que toma a la empresa como el espacio básico a partir del cual desarrollar la homogeneización de los derechos laborales en Europa. Desde esa otra perspectiva, la SE completa, al menos formalmente, un anhelo de cierta forma de ciudadanía que incorpora la noción de democracia industrial à la Marshall como elemento esencial de un derecho ciudadano integral europeo (Marshall, y Bottomore, 1950; Nagels y Sorge, 1977). Las bases del mismo, aunque bajo diferentes articulaciones prácticas, serían compartidas por la mayor parte de países europeos.

Los derechos de ciudadanía adquieren distintas formas en los distintos países. Pero la mayor parte de Estados de Bienestar europeos han pasado a incluir derechos de participación colectiva de los trabajadores en el lugar de trabajo a través de la información, la consulta o la implicación en la toma de decisiones, junto a las correspondientes obligaciones del empleador para respectar dichos derechos y permitir su utilización efectiva (Streeck, 1997: 644).

El aspecto laboral de la SE es una especie de fragmento oculto tras el debate sobre derecho de sociedades que, de manera inmediatamente visible, guía el proceso político de concreción de la normativa. En realidad, es en esta discusión

\footnotetext{
${ }^{5}$ Para otra consideración de la idea de ciudadanía industrial desde la perspectiva de las relaciones laborales europeas ver McGlynn (1995).
} 
subyacente sobre la democracia industrial en Europa donde hay que buscar las causas del enquistamiento de la regulación sobre la SE durante cuarenta años. De hecho, el elemento que mejor explica la larga lista de tentativas de regulación infructuosas sobre esta cuestión es el conflicto abierto en torno a la fórmula de representación de intereses laborales en el consejo de administración de las empresas constituidas como SE.

Todos los proyectos sobre SE presentados por la Comisión Europea, desde el primer borrador de 1966 hasta el texto adoptado en 2001, se enfrentaron a la existencia de visiones muy distintas en torno al significado y extensión de los derechos de representación laboral en la empresa. De un lado, Alemania y Austria, junto a los países nórdicos, mostraron su desconfianza frente a las iniciativas de la Comisión Europea al entender que la normativa laboral asociada a la SE podría debilitar sus sistemas nacionales de representación de trabajadores a escala de empresa, que reconocen extensos derechos de información, consulta y participación. Los sindicatos de estos países presionaron a sus gobiernos para que los proyectos homogeneizadores de la Comisión Europea se inspirasen en sus modelos nacionales de representación y co-determinación. Paradójicamente, en el otro extremo, estas presiones fueron percibidas como un riesgo de desnaturalización por aquellos actores, sobre todo el empresariado, pertenecientes a países en los que la representación laboral a escala de consejo de administración no forma parte del acervo de relaciones laborales. Los empleadores británicos y, en menor medida, los franceses, fueron muy beligerantes ante la importación forzada de prácticas de relaciones laborales que implicaban o bien la introducción de derechos de participación laboral allí donde no existían o bien su ampliación sustancial (Veersma y Swinkels, 2005). Los repetidos bloqueos del gobierno británico a los proyectos de la Comisión Europea o, ya en la década de los noventa, del gobierno español, responden a la presión de estos intereses empresariales.

La posición de la Comisión Europea en este "embrollo de posturas enfrentadas" (Schwinbersky y Gold, 2013: 49) es reveladora de la extrema complejidad del proceso político de gestación de la SE. La cuestión de la SE permanece en la agenda del proyecto comunitario y se reactiva periódicamente por la voluntad política de la Comisión Europea a lo largo de cuatro décadas. Ello no impide que, una y otra vez, se muestre incapaz de conciliar las distintas posiciones de los intereses afectados para llevar adelante su iniciativa de regulación.

Reconstruir el proceso político de la SE es hacer paleontología de la Unión Europea. La discusión sobre la creación de una figura societaria común para los socios de la Comunidad Económica Europea se plantea por vez primera en ámbito público en el $57^{\circ}$ Congreso de Notarios y Registradores Franceses, celebrado en París en 1960 (Blackburn, 1993). La primera iniciativa política sobre esta cuestión se plantea cinco años después por parte del gobierno de Georges Pompidou, que traslada en una nota a sus socios comunitarios la posibilidad de discutir una pieza normativa europea de derecho de sociedades. En 1966, la Comisión Europea hace público un memorando en el que recoge esta idea y comienza a trabajar en una 
primera propuesta de reglamento de SE que ve la luz en 1970 (European Commission, 1970).

El contexto político parecía favorable para la adopción de la iniciativa de la Comisión dado que el objetivo de armonización de la regulación societaria había sido específicamente señalado en las conclusiones del Consejo Europeo de la Haya, celebrado un año antes (Télo y Gobin, 1994). Sin embargo, el texto fue rechazado por el Consejo con una recomendación de revisión. La Tabla 1 enumera los aspectos más ambiciosos (y conflictivos) del primer proyecto de reglamento de SE, presentado en 1970.

\section{Tabla 1. Principales aspectos societarios y laborales del proyecto de reglamento de SE de 1970}

\begin{tabular}{|l|l|}
\hline $\begin{array}{l}\text { Estructura de } \\
\text { gobierno corporativo }\end{array}$ & $\begin{array}{l}\text { Modelo dual, siguiendo el sistema alemán de gobierno } \\
\text { corporativo. Todas las empresas constituidas como SE } \\
\text { combinaría un órgano de dirección ejecutiva y un consejo de } \\
\text { administración, supervisión o vigilancia. }\end{array}$ \\
\hline $\begin{array}{l}\text { Intereses laborales en } \\
\text { órganos de dirección }\end{array}$ & $\begin{array}{l}\text { Reserva de un tercio de los asientos en el consejo de } \\
\text { administración a los representantes de los trabajadores, elegidos } \\
\text { por la plantilla de la empresa. }\end{array}$ \\
\hline $\begin{array}{l}\text { Información, consul- } \\
\text { ta y co- } \\
\text { determinación }\end{array}$ & $\begin{array}{l}\text { Creación de un órgano de información, consulta y co- } \\
\text { determinación laboral en materias específicas de condiciones } \\
\text { laborales, paralelo a la estructura de doble consejo. }\end{array}$ \\
\hline $\begin{array}{l}\text { Negociación } \\
\text { colectiva europea }\end{array}$ & $\begin{array}{l}\text { Reconocimiento del derecho a concluir acuerdos colectivos } \\
\text { europeos sobre las condiciones de empleo en la SE entre la } \\
\text { dirección y los sindicatos representativos, lo que en la práctica } \\
\text { implicaba la creación de un sistema de negociación colectiva } \\
\text { transnacional dentro de la empresa. }\end{array}$ \\
\hline
\end{tabular}

Fuente: adaptado de European Commission (1970).

La nueva versión de reglamento de SE se presentó cinco años más tarde cuando el interés por las cuestiones de armonización societaria se había enfriado, en parte por la incorporación del Reino Unido como nuevo socio en 1973 (European Commission, 1975). El principal cambio introducido afectó al criterio de distribución de los asientos entre representantes de los accionistas y de los empleados en el consejo de administración, con la siguiente propuesta: reserva de un primer tercio de los consejeros para los accionistas; de un segundo tercio para los empleados; y de un último tercio según elección conjunta de ambos colectivos.

La reactivación de la iniciativa normativa sobre la SE esperó a un nuevo momento político favorable a finales de la década de los ochenta. En 1988, la Comisión Europea presentó un Memorando que insistía en la necesidad de desarrollar instrumentos societarios coherentes con el Mercado Único, establecido tras la aprobación del Acta Única Europea de 1986 (European Commission, 1988). El Memorando reactivó la cuestión de la SE en el contexto de los debates sobre el mercado interior de libre circulación de mercancías, servicios y capitales. En una 
muestra de posibilismo, la Comisión renunció a la idea de imponer un solo modelo de implicación laboral en la SE y, en 1989, presentó las iniciativas de regulación societaria y laboral en dos piezas legales separadas, para que la previsible controversia sobre la segunda no bloquease las negociaciones sobre la primera. A pesar de ello, la propuesta de reglamento de SE fue nuevamente rechazada a instancias del Reino Unido (European Commission, 1989).

La Tabla 2 resume los principales aspectos de las propuestas de Estatuto y Directiva presentados por la Comisión Europea en 1989, menos ambiciosos en sus objetivos de armonización legal que las iniciativas de la década de los setenta.

Tabla 2. Principales aspectos societarios y laborales del proyecto de reglamento de SE y de Directiva de implicación de empleados asociada de 1989

\begin{tabular}{|l|l|}
\hline $\begin{array}{l}\text { Estructura de } \\
\text { gobierno corporativo }\end{array}$ & $\begin{array}{l}\text { Posibilidad de escoger entre estructura de consejo monista o } \\
\text { dual. }\end{array}$ \\
\hline $\begin{array}{l}\text { Intereses laborales } \\
\text { en órganos de } \\
\text { dirección }\end{array}$ & $\begin{array}{l}\text { Posibilidad de escoger entre cuatro modelos diferentes de } \\
\text { implicación laboral en la SE, con un perímetro distinto de } \\
\text { derechos y obligaciones inspirado en los sistemas de } \\
\text { representación de trabajadores alemán, nórdico, francés y } \\
\text { holandés. }\end{array}$ \\
\hline $\begin{array}{l}\text { Discrecionalidad de } \\
\text { la dirección }\end{array}$ & $\begin{array}{l}\text { En caso de imposibilidad de alcanzar un acuerdo en torno al } \\
\text { modelo de representación laboral en la SE, se aplicará el criterio } \\
\text { de la dirección de la empresa. }\end{array}$ \\
\hline $\begin{array}{l}\text { Flexibilidad de la } \\
\text { transposición } \\
\text { nacional }\end{array}$ & $\begin{array}{l}\text { En aplicación del principio de subsidiariedad, los Estados } \\
\text { miembros podrían excluir alguno de los modelos de } \\
\text { representación laboral en la SE durante el proceso de } \\
\text { transposición nacional de la normativa comunitaria. }\end{array}$ \\
\hline
\end{tabular}

Fuente: adaptado de European Commission (1989).

La Comisión lo intentó dos años después, a través de una nueva versión revisada de reglamento de SE, también acompañada de Directiva de participación laboral (European Commission, 1991a; 1991b). Sin embargo, como en las ocasiones anteriores, la tentativa resultó bloqueada ante la imposibilidad de obtener el acuerdo unánime del Consejo (Blackburn, 1993). La versión de 1991 redujo aún más los umbrales de armonización y ofreció una mayor flexibilidad para adaptar la regulación a las especificidades societarias y laborales de los Estados miembros. La nueva rebaja de exigencias se expresó en los siguientes términos: los Estados miembros podían fijar el modelo de gobierno corporativo, monista o dual, que considerasen oportuno en la transposición nacional de la norma europea; (2) en caso de ausencia de acuerdo entre dirección y empleados acerca del modelo de representación laboral, sería la Junta de Accionistas quien determinase el modelo laboral a seguir de entre los recogidos en la transposición nacional (European Commission, 1991a; 1991b).

Pese a acumular un nuevo fracaso, la segunda oleada de esfuerzos sobre la SE dejó en la agenda comunitaria una huella más profunda que la primera. El bloqueo 
de las iniciativas de 1989 y 1991 no fue tomado como una derrota por la Comisión Europea. Se interpretó, más bien, como un episodio de adquisición de experiencia política que, una vez retirado el escollo de la exigencia de unanimidad de voto en el Consejo para la adopción de normativa comunitaria, permitiría adoptar la regulación sobre la SE. Tras el Tratado de Maastricht, desapareció esta exigencia de unanimidad, lo cual permitió a la Comisión Europea alcanzar su primer gran éxito en materia de regulación laboral europea con la Directiva sobre comités de empresa europeos (González Begega, 2011) .

El camino quedó expedito en cuanto a exigencias procedimentales, pero la adopción de la regulación sobre la SE aún debió esperar casi una década. La Comisión encargó un informe sobre el Estatuto de SE a un grupo de expertos presidido por Etienne Davignon. El informe fue presentado a los comisarios Monti, del Directorado General (DG) de Mercado Interno y Servicios, y Flynn, del DG de Relaciones laborales, Empleo y Asunto sociales, a mediados de 1997 (High Level Group on Workers' Involvement, 1997). El documento recomendaba una nueva rebaja de las exigencias de armonización legal para salvar el problema de bloqueo político. El informe Davignon reconocía la imposibilidad de resolver la heterogeneidad de los modelos de representación de trabajadores a escala de empresa en Europa. Dado lo inasequible del objetivo de homogeneización, proponía una Directiva de implicación laboral en la SE que apostase por una opción de negociación de los términos de participación de los trabajadores por las partes y que reconociese la diversidad de tradiciones y modelos de representación laboral en órganos de dirección corporativa de los Estados miembros (Conchon, 2011; Conchon y Waddington, 2011). En caso de fracaso de esta primera vía negociada, sería aplicable un conjunto de reglas de implicación laboral estándar y de mínimos (Keller y Werner, 2012).

La Comisión Europea recogió la apuesta del Informe Davignon por una opción de contenidos aceptables para todos. La regulación sobre la SE fue finalmente adoptada en la reunión del Consejo Europeo de Niza, en diciembre de 2000. A partir de ese momento, se abrió un plazo de cuatro años, hasta octubre de 2004, para que los Estados miembros completasen el proceso de transposición nacional.

\footnotetext{
${ }^{6}$ Directiva 94/45/CE del Consejo de 22 de septiembre de 1994, sobre la constitución de un comité de empresa europeo o de un procedimiento de información y consulta a los trabajadores en las empresas y grupos de empresas de dimensión comunitaria, OJ L 254, 30/09/1994. La Directiva 94/45/CE también resultó bloqueada durante dos décadas por la necesidad de superar el condicionamiento de unanimidad de voto en el Consejo, según el Artículo 100 del Tratado de Roma. Finalmente fue adoptada el 22/09/1994 aplicando el procedimiento del artículo 2.2. del Protocolo Social anexo al Tratado de Maastricht.
} 


\section{La Sociedad Europea y su Directiva de implicación laboral. Principales aspectos normativos}

La normativa sobre la SE confiere un impulso al proceso de europeización de las regulación sobre sociedades y de las relaciones laborales. Pese a sus limitaciones, introduce un sistema de implicación de trabajadores en los consejos de administración de las empresas que, voluntariamente, se constituyan como SE. Los reglamentos de la Sociedad Anónima Europea y de la Sociedad Cooperativa Europea y sus Directivas asociadas constituyen un logro, más allá de su ductilidad. En cualquier caso, antes que una regulación europea propiamente dicha, la normativa sobre la SE es "una plantilla" a partir de la que se despliega una compleja y diversa regulación nacional (Fulton, 2013).

El reglamento de la SE recoge las cuestiones relativas a la fórmula de constitución y estructura de las empresas constituidas bajo esta forma societaria. La ductilidad del texto permite su adaptación a la heterogeneidad del derecho de sociedades y laboral en Europa. Asimismo, atendiendo a las recomendaciones del Informe Davignon (High Level Group on Workers' Involvement, 1997), se apuesta por el principio de subsidiariedad, permitiendo que sean las partes interesadas en el ámbito de la empresa quienes, a través de la negociación, se hagan responsables del modelo específico de participación laboral en la SE.

El articulado de la regulación europea sobre la SE presenta vacíos intencionales. El objetivo es que estos vacíos sean cubiertos a través de los procesos de transposición nacional de la normativa y de negociación a escala de empresa (Esteban Velasco y Fernández del Pozo, 2004). La norma a la carta que consolida este modelo de regulación europea implica una renuncia a cualquier horizonte de armonización (Keller, 2002). El texto de mínimos impulsado por la Comisión Europea consiguió superar el bloqueo político, pero, al mismo tiempo, ha limitado la eficacia de la regulación sobre la SE en la mayor parte de Estados miembros (González Begega y Köhler, 2012).

En términos de derecho de sociedades, la SE aspira a convertirse en un instrumento de facilitado de las operaciones empresariales transfronterizas en Europa, incluyendo fusiones y adquisiciones. La SE ofrece a las empresas con domicilio social en la Unión Europea, además de Noruega, Islandia y Liechtenstein, una forma de derecho de sociedades para la constitución de nuevas empresas o la transformación de empresas ya existentes. Todas las empresas con operaciones en dos o más Estados miembros pueden acogerse voluntariamente a la forma de SE sin restricciones adicionales de localización geográfica o volumen de empleo. El reglamento sobre la SE presenta omisiones conscientes dentro de su articulado en materia de fiscalidad, competencia o quiebra, siendo la intención del regulador que dichos vacíos puedan ser cubiertos en la transposición nacional. Para Stollt y Kluge (2011), la normativa sobre la SE es una forma híbrida de regulación a doble nivel, que ha tenido como resultado la entrada en vigor de una gran número de piezas legales sobre la SE a escala nacional. La proximidad entre unas y otras no responde a la capacidad armonizadora de la regulación comunitaria sino a la existencia de 
pautas afines de derecho societario y laboral entre los Estados miembros. Así, la SE no constituye una forma completa de regulación europea sino más bien una especie de plantilla remitida a los dominios del legislador nacional para su adaptación. Las empresas constituidas como SE se ven favorecidas por esta flexibilidad, pudiendo escoger el marco nacional de regulación sobre la SE que más se ajuste a sus intereses, contemplándose además posibilidades de transferencia de domicilio social y cambio de regulación nacional.

\section{Tabla 3. La SE. Cobertura, constitución y estructura de gobierno corporativo}

\begin{tabular}{|l|l|}
\hline Empresas cubiertas & $\begin{array}{l}\text { Empresas con actividades a escala europea (operaciones en, } \\
\text { al menos, dos Estados dentro la Unión Europea, Noruega, } \\
\text { Islandia y Liechtenstein) que deseen constituirse como SE. }\end{array}$ \\
\hline Iniciativa de constitución & $\begin{array}{l}\text { Creación de SE voluntaria. Discrecional por parte de la } \\
\text { empresa. }\end{array}$ \\
\hline $\begin{array}{l}\text { Estructura de gobierno } \\
\text { corporativo }\end{array}$ & $\begin{array}{l}\text { Posibilidad de escoger entre estructura de consejo monista o } \\
\text { dual, según la transposición nacional. }\end{array}$ \\
\hline
\end{tabular}

Fuente: Reglamentos del Consejo de Europa (CE) 2157/2001 y 1435/2003.

En materia laboral, la SE ofrece una maleabilidad similar (Leca, 2007). Las Directivas que acompañan a los Estatutos de SE y de SCE establecen un doble canal para la representación de los empleados, que en todo caso se verá sujeto a la negociación en el ámbito de la empresa ${ }^{7}$.

En primer lugar, se prevé la creación de un órgano de representación laboral o comité de empresa de la SE, con competencias de información y consulta sobre aspectos de carácter transnacional relacionados con operaciones y empleo que afecten a la sociedad o a alguna de sus filiales o establecimientos. Dicho órgano deberá reunirse al menos una vez al año con la dirección ${ }^{8}$.

En segundo lugar, se recoge el derecho de representación de los trabajadores en el máximo órgano de gobierno corporativo de la sociedad. Al igual que en el caso anterior, no obstante, el reconocimiento de este derecho queda supeditado a su recogida por la transposición nacional del texto comunitario y a la negociación del

${ }^{7}$ Las disposiciones de referencia de la Directiva del Consejo de Europa 2001/86/CE sobre las fórmulas de implicación de trabajadores en la SE, recogidas en su Anexo, son efectivas únicamente en caso de fallo de las negociaciones entre dirección y representantes de los empleados, en virtud de lo contemplado por el artículo 7.

${ }^{8}$ Artículos 2(i) y 2(j) de la Directiva del Consejo de Europa 2001/86/CE. Anexo de la Directiva 2001/86/CE, Disposiciones de referencia, Partes 1 y 2. 
mismo en el ámbito de la empresa antes de que se tramite el registro de la $\mathrm{SE}^{9}$. La Tabla 4 recoge los principales aspectos de las Directivas sobre implicación laboral en la SE.

\section{Tabla 4. Principales aspectos de la implicación laboral en la SE}

\begin{tabular}{|l|l|}
\hline $\begin{array}{l}\text { Derechos de } \\
\text { representación }\end{array}$ & $\begin{array}{l}\text { (1) Comité de empresa de la SE (órgano de información y } \\
\text { consulta a los empleados). } \\
\text { (2) Representación en el consejo de administración de la } \\
\text { empresa (cuando sea aplicable y siempre condicionado a su } \\
\text { reconocimiento en la transposición al marco legal nacional). }\end{array}$ \\
\hline $\begin{array}{l}\text { Procedimiento de } \\
\text { negociación }\end{array}$ & $\begin{array}{l}\text { Acuerdo entre la dirección y un grupo especial de } \\
\text { negociación o aplicación de las disposiciones estándar } \\
\text { recogidas en el anexo de la Directiva (tras 6 meses / 1 año de } \\
\text { plazo). }\end{array}$ \\
\hline
\end{tabular}

Fuente: Directivas 2001/86/CE y 2003/72/CE.

La negociación de la fórmula de implicación laboral en la SE es una de las cuestiones en las que la estrategia de regulación dúctil de la Comisión Europea se muestra con más claridad. La determinación del modelo de implicación de trabajadores en el consejo de administración de la SE resulta de la negociación entre la dirección de la empresa y un grupo especial de negociación formado por trabajadores creado a tal efecto. El reglamento de la SE señala que la empresa no podrá ser registrada como SE hasta que no se haya llegado a un acuerdo sobre la implicación de trabajadores.

Las negociaciones pueden arrojar tres resultados: las dos partes firman un acuerdo de participación de trabajadores en la futura SE; se aplican las normas estándar recogidas en el anexo de la Directiva; o el grupo especial de negociación decide no abrir las negociaciones o las da por finalizadas (Stollt y Kluge, 2011a: 72).

Así, la negociación de la implicación de trabajadores en la SE ofrece importantes similitudes con el procedimiento establecido para la negociación de los comités de empresa europeos: se prioriza cualquier acuerdo que las partes puedan llegar a alcanzar dentro del ámbito de la empresa y la Directiva únicamente proporciona un soporte de mínimos en caso de fracaso de las negociaciones. La principal diferencia con la negociación de un comité de empresa europeo es que la iniciativa emana de la dirección de la empresa y no de los trabajadores (Kluge y Stollt, 2007). Asimismo, la duración máxima del proceso de negociación es de seis meses, ampliable hasta un año si las partes así lo convienen.

\footnotetext{
${ }^{9}$ Artículo 2(k) de la Directiva del Consejo de Europa 2001/86/CE. Anexo de la Directiva 2001/86/CE, Disposiciones de referencia, Parte 3.
} 
La SE extiende la arquitectura de derechos de participación de los trabajadores europeos más allá de la información y consulta de los comités de empresa europeos, que se replican en el comité de empresa de la SE. Para ello, articula un mecanismo de participación laboral en la toma de decisiones corporativas a escala transnacional, condicionado a la negociación y a las limitaciones establecidas por las transposiciones nacionales (Gold, Nikopoulos y Kluge, 2009).

Tal y como señala Davies (2003), este último es, seguramente, el principal constreñimiento estructural de la normativa sobre la SE, al consolidar un modelo de participación atrapado por las trayectorias previas de representación de empleados a escala nacional, "sin escapatoria ni posibilidades de exportación”.

En realidad, no existe una sola normativa sobre la SE sino un conjunto de variantes nacionales que reproducen las expectativas, usos y prejuicios culturales y políticos de las distintas tradiciones de relaciones laborales sobre la participación de empleados en la empresa (Taylor, 2006). La transposición española de la normativa sobre la SE es un buen ejemplo de ello.

En España, la transposición del aparato normativo sobre la SE se vio precedida de un intenso debate parlamentario que dilató la culminación del proceso más allá del calendario previsto ${ }^{10}$. Los aspectos más controvertidos de la transposición, que fue aprobada con los votos en contra del Partido Popular, hicieron referencia al procedimiento de designación de los miembros en la comisión negociadora, la participación de representantes sindicales, la distribución de asientos reservados a los trabajadores en el consejo de administración, la financiación de expertos y el presupuesto de los representantes de los trabajadores, incluyendo el crédito de horas anuales retribuidas para el ejercicio de sus funciones, el procedimiento de renegociación del acuerdo en caso de alteración de la estructura de la empresa y la introducción de cláusulas específicas de confidencialidad para los miembros laborales del consejo de administración de la SE (Esteban Velasco, 2007; Valdés Dal-Re, 2007). El aspecto más interesante de la transposición de la SE desde un punto de vista societario es la posibilidad de que las SE domiciliadas en España puedan escoger entre los modelos monista y dual de gobierno corporativo, introduciéndose el segundo como novedad en el marco español de derecho de sociedades.

La pérdida de impacto producida por esta desagregación normativa es uno de los aspectos discutidos en un informe de evaluación de la regulación sobre la SE

${ }^{10}$ Ley 19/2005, de 14 de noviembre, sobre la sociedad anónima europea domiciliada en España (BOE número 273 de 15/11/2005, páginas 37303 a 37308, referencia BOE-A-200518667); Ley 31/2006, de 18 de octubre, sobre implicación de los trabajadores en las sociedades anónimas y cooperativas europeas (BOE número 205 de 19/10/2006, páginas 36302 a 36317, referencia BOE-A-2006-18204); Ley 3/2011, de 4 de marzo, por la que se regula la Sociedad Cooperativa Europea con domicilio en España (BOE número 57 de 8/03/2011, páginas 25803 a 25809, referencia BOE-A-2011-4288). 
encargado por la Comisión Europea a la consultora Ernst \& Young, que resultó altamente polémico al sugerir que la implicación de trabajadores constituía una 'carga' para el adecuado desarrollo de la SE (Ernst \& Young, 2009). El documento fue duramente criticado desde posiciones sindicales (ETUI, 2010). En cualquier caso, en julio de 2011, y como prueba tácita de insatisfacción, el DG de Empleo, Asuntos Sociales e Inclusión de la Comisión decidió emprender una ronda de consultas con los agentes sociales europeos sobre la eventual revisión de la Directiva sobre implicación de trabajadores en la SE (European Commission, 2011).

Las respuestas encontradas de la patronal BusinessEurope y de la Confederación Europea de Sindicatos (CES), emitidas en octubre de 2011, explican la paralización de la iniciativa. BusinessEurope se mostró contraria a la modificación de la Directiva y sugirió una simplificación de las reglas societarias de la SE (BusinessEurope, 2011). Por su parte, la CES exigió la apertura de proceso de revisión, manifestándose frustrada por el desarrollo práctico de la SE e identificando tres áreas problemáticas de la implicación de trabajadores: (1) la complejidad del procedimiento de implicación laboral; (2) la ausencia de garantía legal en el procedimiento de negociación; y (3) la preocupación de que algunas empresas pudieran hacer uso de la SE para rebajar los derechos de implicación de los trabajadores en aquellos países con sistemas de participación laboral más desarrollados. La CES insistió en que cualquier iniciativa de revisión de la Directiva sobre implicación laboral en la SE debería implicar, asimismo, la apertura del procedimiento de revisión del reglamento sobre la SE, al no ser posible considerar ambas piezas legales por separado (ETUC, 2011).

El proceso de revisión de la Directiva sobre implicación laboral en la SE se encuentra paralizado. La Comisión Europea considera que el principal problema en torno al bajo interés suscitado por la SE no estriba tanto en posibles deficiencias de la regulación como en la falta de información disponible por las empresas que podrían estar interesadas en hacer uso de este instrumento de constitución de sociedades. De hecho, el último Plan de Acción de la Comisión Europea sobre derecho de sociedades y gobierno corporativo elude referirse a la revisión de la regulación sobre la SE, recomendando en su lugar el desarrollo de:

Un esfuerzo para incrementar el conocimiento de [la regulación entre las empresas] (...) a través de una campaña de información sobre el Estatuto de SE que contará con el soporte de una página electrónica en la que se recogerán información práctica y documentos relevantes sobre el mismo (European Commission, 2012: 14) ${ }^{11}$.

${ }^{11}$ El DG de Mercado y Servicios de la Comisión Europea ha desarrollado una página electrónica sobre la SE que estará disponible para consulta pública a mediados de noviembre de 2013. Inicialmente se ofrecerá en tres idiomas: inglés, francés y alemán. La página electrónica tiene como objetivo incrementar la información disponible sobre procedimiento de constitución y funcionamiento de la SE, incluyendo una recopilación de las transposiciones nacionales sobre la SE, así como otras piezas legales de derecho de 


\section{La Sociedad Europea. Variedades operativas y despliegue práctico}

El número de empresas constituidas como SE a 1 de Enero de 2013 se situaba en 1601 , según datos de la Red SEEurope ${ }^{12}$. Detrás de la cifra se oculta una realidad compleja de formas operativas de SE. Además, el impacto de la regulación sobre la SE resulta altamente desigual en términos geográficos. Frente a la marginalidad del proceso en el Sur de Europa, la SE como forma societaria ha suscitado un mayor interés en otros países, como Alemania, y también, aunque por razones distintas, en la República Checa.

Tal y como señalan Rehfeldt et al. (2011), la diversidad de realidades societarias y laborales que encubre la SE es muy amplia. En muchos casos, las SE constituidas son, en realidad, empresas sin actividad o sin trabajadores. De las 1601 SE identificadas en Enero de 2013, solamente en 234 había logrado constatarse la existencia de actividad económica y la presencia de una plantilla de, al menos, cinco trabajadores.

A pesar de ello, es necesario reconocer que la investigación empírica sobre el desarrollo de las SE aún no se encuentra demasiado desarrollada. Existe, eso sí, un número reducido pero altamente interesante de estudios de caso sobre procesos de negociación en empresas individuales (Knudsen, Müller y Rehfeldt, 2008), análisis comparados sobre el rendimiento la participación laboral en diferentes SE (Rehfeldt et al., 2011) y evaluaciones sobre el impacto de la introducción de la normativa sobre la SE a escala nacional (Veersma y Swinkels, 2005; Cremers, Stollt y Vitols, 2013).

En todo caso, la evidencia sobre el funcionamiento práctico de las SE debe aún ampliarse para comprender por qué en muchos casos la creación de la SE responde a operaciones estrictamente societarias de facilitado de adquisiciones, tomas de control y movilidad transfronteriza de sociedades, sin consecuencias efectivas sobre la participación de trabajadores a escala transnacional. Pese a este déficit, Rehfeldt

sociedades y laboral nacional relevantes para su funcionamiento. Asimismo se incorporará un protocolo de registro en línea voluntario para las nuevas SE constituidas.

${ }^{12}$ No existe oficina de registro europea sobre la SE, aunque el Suplemento al Diario Oficial de la Unión Europea (TED) publica regularmente información sobre la constitución de nuevas SE. En ausencia de registro central, y dada la diversidad de fórmulas de registro de sociedades a escala nacional, resulta altamente complejo trazar un mapa actualizado del número de SE activas. La Red SEEurope, dependiente del European Trade Union Institute (ETUI), lleva recopilando información sobre esta cuestión desde 2005. Su base de datos, European Company Database (ECDB: http://ecdb.worker-participation.eu) identifica aproximadamente un $60 \%$ más de SE activas de las recogidas por el TED y, a expensas del desarrollo que pueda tener la página electrónica de la Comisión Europea (ver nota anterior), se ha convertido en la principal fuente de información sobre la SE. 
et al. (2011) proponen una tipología de SE en función de su actividad y volumen de empleo, distinguiendo entre SE normales, micro, vacías y ovni.

La Tabla 5 recoge la descripción de estas categorías de SE así como el número de empresas identificadas para cada una de ellas.

Tabla 5. Categorías de SE según su actividad y volumen de empleo

\begin{tabular}{|l|l|l|}
\hline CATEGORÍA & DESCRIPCIÓN & NÚMERO \\
\hline SE normal & Con actividad y más de cinco empleados & 234 \\
\cline { 1 - 2 } Micro-SE & $\begin{array}{c}\text { Con actividad y con cinco o menos emplea- } \\
\text { dos }\end{array}$ & \multirow{2}{*}{256} \\
\hline SE vacía & $\begin{array}{l}\text { Con algún tipo de actividad, pero sin em- } \\
\text { pleados }\end{array}$ & \multirow{2}{*}{$1111^{13}$} \\
\hline SE ovni & $\begin{array}{l}\text { Identificadas pero aún no asignadas a una } \\
\text { categoría estable por falta de información }\end{array}$ \\
\hline
\end{tabular}

Fuente: ECDB (1/01/2013).

El impacto geográfico de la SE es muy desigual. Como muestra el Gráfico 1, la mayor parte de países cubiertos mantienen una relación marginal con esta forma societaria. Solo Holanda, Eslovaquia, Reino Unido, Alemania y la República Checa superan el número de treinta SE constituidas. La marginalidad del proceso resulta especialmente llamativa en el caso Francia o los países Nórdicos, con un importante acervo de transnacionalización corporativa y amplia experiencia en otros procesos de las relaciones laborales europeas, como los comités de empresa europeos.

${ }^{13} 909$ de estas 1111 SE caracterizadas como ovni tienen domicilio social en la República Checa. Se carece de datos sobre actividad y volumen de empleo acerca de ellas. 


\section{Gráfico 1. SE activas según contenido y país de domicilio social}

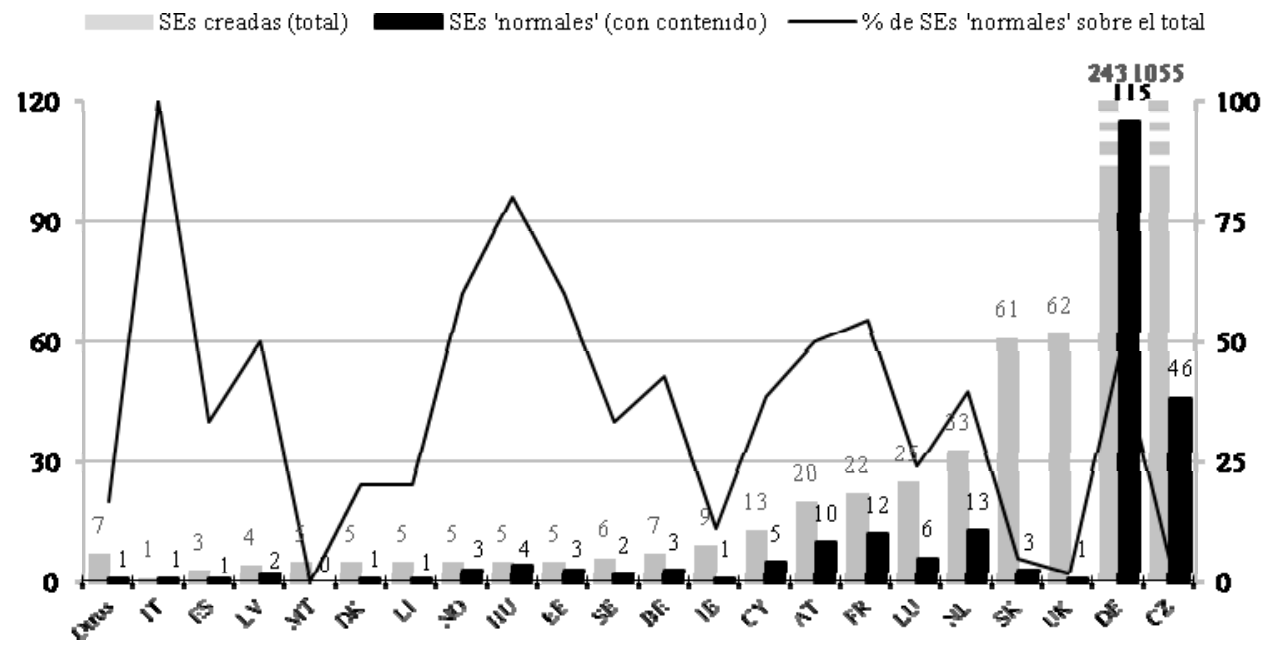

Fuente: ECDB (1/01/2013).

En términos cuantitativos, la intensidad del proceso de creación de SE en Alemania, la República Checa enmascara el impacto real de la normativa sobre la SE a escala europea. El significado del proceso tampoco es similar en estos dos países, que suman el 81,07\% de las SE constituidas en Enero de 2013. La relación entre SE normales y atípicas ha generado un interesante debate acerca de las razones de la concentración de SE atípicas en la República Checa frente al más regular porcentaje de SE normales en Alemania. Tal y como indica Köstler (2013), el hecho de que Alemania concentre casi la mitad de las SE con contenido (un 49,14\%) era algo esperable al inicio del proceso, dado el fácil acomodo de la tradición de participación laboral alemana al modelo de la SE (ver también, Gold y Schwimbersky, 2008).

Por su parte, Eidenmüller y Lasak (2011) abordan el "puzle de la SE en la República Checa" e indican que la preferencia de muchas empresas por la transposición checa de la normativa sobre la SE va mucho más allá de los objetivos de simplificación de la estructura de gobierno corporativo, de reducción del número de integrantes del consejo de administración o de la imagen positiva de la SE en este país como vehículo de movilidad societaria transnacional, factores todos ellos a los que aludía el informe sobre impacto práctico de la SE elaborado por Ernst \& Young (2009).

¿Por qué tantas SE en la República Checa no tienen actividad o son descritas como ovni en la tipología del ETUI? Una explicación se refiere a las cuestiones de implicación de trabajadores y transferencia de domicilio social. No existe necesidad legal de llevar a cabo negociaciones sobre participación laboral en la SE si esta (...) 
no tiene empleados. La regulación establece que dichas negociaciones deberán tener lugar más tarde, una vez la SE vacía sea activada - una vez comienza a tener actividades y a contratar empleados. No obstante, no se establecen claramente las circunstancias bajo las cuales se entiende que una SE ha sido activada. Además, se han detectado casos en otros Estados miembros, como Alemania, en los que (...) la SE no tiene empleados y, previsiblemente, no los tendrá en el futuro. Las oficinas de registro sospechan que ésta puede ser una estrategia para eludir las provisiones de participación de empleados en la SE (Eidenmüller y Lasak, 2011: 12).

En realidad, lo que encubre la extensión de la SE en la República Checa es la estrategia de algunas empresas para aprovechar un resquicio legal de la normativa y soslayar los derechos de representación de trabajadores que ésta recoge. Para determinados empleadores, la opción checa abre la posibilidad de crear una SE evitando todo tipo de obligación laboral y esquivar, por ejemplo, los derechos de participación que establecen las regulaciones alemana o austriaca (Cremers y Carlson, 2013).

La SE en España, como en otros países del Sur de Europa ha tenido un desarrollo práctico marginal. La Tabla 6 recoge los detalles de las SE registradas en España hasta Junio de 2013. De las tres SE activas en Enero de 2013, solamente se disponían de datos que confirmaban su carácter de SE normal para una de ellas. En Marzo de 2013 se produjo una transferencia de domicilio social de una nueva SE desde Alemania, también con actividad y más de cinco empleados.

Tabla 6. La SE en España

\begin{tabular}{|l|l|l|l|l|}
\hline EMPRESA & REGLAMENTO & REGISTRO & ESTADO & CATEGORÍA \\
\hline $\begin{array}{l}\text { Arcelor Steel } \\
\text { Trade SE }\end{array}$ & SE & $14 / 07 / 2008$ & $\begin{array}{l}\text { Disuelta } \\
(19 / 01 / 2011)\end{array}$ & Vacía \\
\hline $\begin{array}{l}\text { Paypal SE Sucusal } \\
\text { in Spain }\end{array}$ & SE & $22 / 04 / 2009$ & Activa & Ovni \\
\hline $\begin{array}{l}\text { Euskal Herriko } \\
\text { Ikastolak }\end{array}$ & SCE & $02 / 12 / 2009$ & Activa & Normal \\
\hline $\begin{array}{l}\text { Agence Générale } \\
\text { de Marques et de } \\
\text { Brevets SE }\end{array}$ & SE & $16 / 07 / 2010$ & Activa & Ovni \\
\hline Ortus Casa SE & SE & $13 / 03 / 2013$ & Activa & Normal \\
\hline
\end{tabular}

Fuente: Registro Mercantil Central (1/07/2013).

González Begega y Köhler (2012) han evaluado las causas de la escasa atención prestada al proceso de la SE en España. Tal y como ocurre con la transposición nacional de la normativa sobre la SE en otros países del Sur de Europa donde existe un importante acervo experiencias de transnacionalización de intereses corporativos, empresas y agentes sociales han mostrado un muy bajo grado de interés en este fórmula societaria (Menjucq, Fages y Vuidard, 2008). En España, los expertos de relaciones laborales internacionales de la CEOE y de los sindicatos 
mayoritarios, UGT y CCOO, coinciden al apuntar hacia la inexistencia de incentivos fiscales, la complejidad de la normativa y la introducción de elementos ajenos a la práctica nacional de relaciones laborales como las principales causas del escaso interés de las empresas españolas hacia la SE. El desconocimiento de la norma, las dificultades técnicas y la percepción de potenciales recortes en la discrecionalidad empresarial dentro de la toma de decisiones corporativas habrían desincentivado la extensión de esta forma societaria. Ante lo limitado de su impacto, las organizaciones sindicales apenas han prestado atención a la SE, a pesar de que potencialmente introduce un nuevo paradigma de representación de intereses laborales a escala de empresa en España. Las razones de este desinterés estriban en una cuestión práctica. Dado que la constitución de la SE depende de la voluntad del empleador y éste, en España, no se ha mostrado interesado por esta forma societaria, constituiría una pérdida de tiempo invertir esfuerzo en un proceso que carece de desarrollo real. Los sindicatos, de hecho, carecen de un información acerca de los representantes de trabajadores españoles que pudieran formar parte de un comité de empresa de SE o que se encontrasen en disposición de ser consejeros en un consejo de administración de $\mathrm{SE}^{14}$.

Otro aspecto importante es la secuencia de constitución de nuevas SE a lo largo del periodo 2004-2013, que recoge el Gráfico 2. El ritmo de creación de SE experimenta un fuerte crecimiento a partir de 2007, que se encuentra relacionado con incorporación de las SE registradas en la República Checa y que distorsiona la relación entre SE normales y atípicas. Si en 2007 el porcentaje de SE con contenido alcanzaba el 30,43\% de las totales, en Enero de 2013 el porcentaje se ha visto reducido al $14,61 \%$.

${ }^{14}$ La ECDB (http://ecdb.worker-participation.eu) no identifica ningún representante laboral en consejo de administración de SE de nacionalidad española a fecha de 1 de enero de 2013. 


\section{Gráfico 2. Secuencia de creación de SE (2004-2013).}

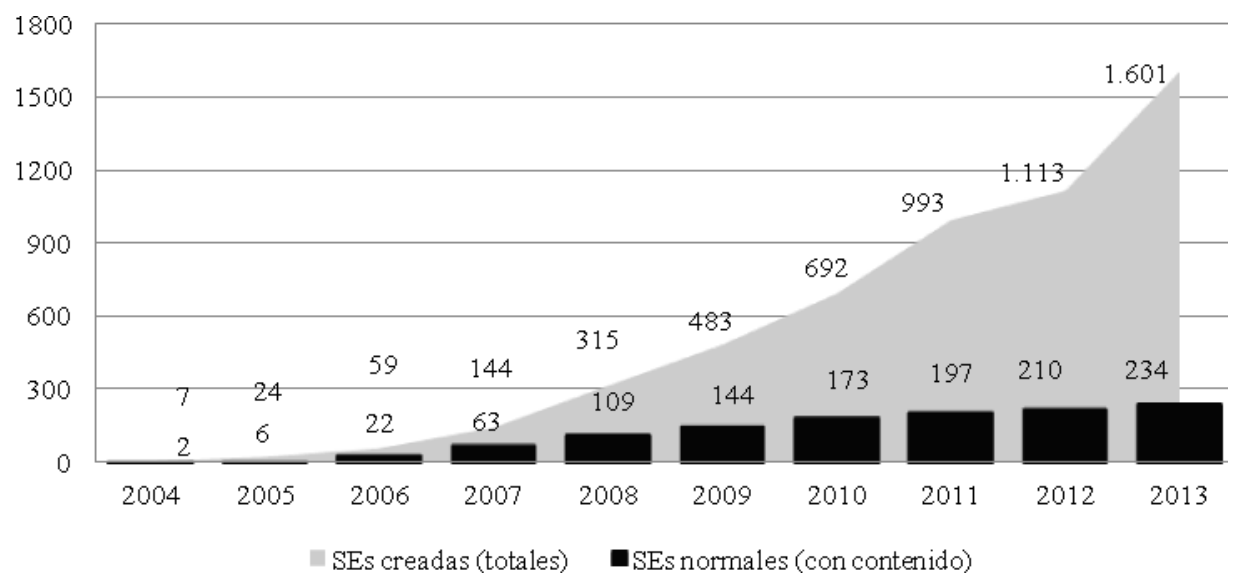

Fuente: ECDB (1/01/2013).

No obstante, el aspecto de impacto en el que se puede observar más nítidamente las limitaciones de la SE, al menos desde una perspectiva laboral, es su escasa capacidad de dinamización de las prácticas de implicación de trabajadores en la empresa. El impacto transformador de la SE sobre las relaciones laborales europeas resulta extraordinariamente tímido. Del total de 1601 SE constituidas en Enero de 2013 solamente en 88 de ellas (un 5,49\%) era posible identificar la existencia de una práctica de representación de trabajadores según lo establecido por la Directiva de implicación laboral de la SE.

Dentro de estas 88 empresas, correspondientes a SE con actividad y empleo, 42 contaban con un procedimiento de información y consulta a los trabajadores a través de un comité de empresa de la SE, pero solo 46 suplementaban este órgano con una práctica estable de representación de intereses laborales en el consejo de administración de la SE. La participación en la toma corporativa de decisiones a escala transnacional únicamente queda asegurada en un 2,78\% de las SE registradas. Además, 37 de estas 46 SE tenían, a comienzos de 2013, su domicilio social en Alemania. Atendiendo a las particularidades del sistema alemán de codeterminación (Köhler, 2013), la SE no habría aportado una novedad significativa de participación para los empleados domésticos de estas empresas. Si lo habría hecho, no obstante, para un número de trabajadores a operaciones de la SE en otros países que se habrían visto ampliados sus derechos de representación laboral ${ }^{15}$.

15 La ECDB identificaba 140 representantes laborales procedentes de doce países distintos formando parte de consejos de administración de SE en igualdad de obligaciones y derechos que los representantes de los accionistas, a fecha de 1 de enero de 2013. La 
Al constatar estos datos, tal vez resulte oportuno (por irónico) citar el contenido del considerando 6 de la Directiva 2001/86/CE, sobre implicación de trabajadores en la SE: "en todos los casos de constitución de SE deberán asegurarse los procedimientos de información y consulta a escala transnacional”. El Gráfico 3 recoge los datos de la implicación de trabajadores en la SE.

\section{Gráfico 3. La implicación de trabajadores en la SE}

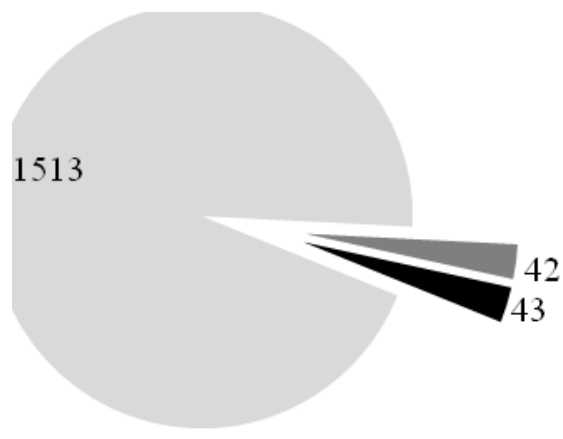

SE sin prácticas de implicación lab oral (vacías, micro y ovni)

- SE con informacióny consulta (normales)

- SE con información, consultay participación (normales)

Fuente: ECDB (1/01/2013).

\section{Conclusiones. ¿Motivos para la frustración? El largo impasse de las relaciones laborales europeas}

El desenvolvimiento práctico de la SE cuestiona las expectativas suscitadas con motivo de la adopción de la normativa. Del juicio optimista de algunos expertos (Lenoir, 2007) en torno al potencial transformador de la SE para las relaciones laborales de empresa europeas se ha pasado a un escenario en el que resultan dominantes otras valoraciones más cautas. La SE sigue teniendo potencial para dar un impulso a la democracia industrial en Europa pero, por motivos normativos y de aplicación práctica, constituye un instrumento sin desarrollar (Keller y Werner, 2012).

En primer lugar, la ductilidad de la orientación legal europea, en un camino de regulación laboral flexible abierto por la normativa sobre comités de empresa

procedencia nacional de estos consejeros era la siguiente: Alemania, Bélgica, República Checa, Dinamarca, Francia, Hungría, Italia, Holanda, Noruega, Polonia, Reino Unido. En este sentido, la principal innovación introducida por la SE es el carácter transnacional adquirido por los derechos de implicación, dado que en la lista se incluyen nacionales de países para los cuales el reconocimiento de la representación laboral en la toma de decisiones corporativas resulta ajeno a la tradición de relaciones laborales. 
europeos, ha derivado en una fórmula no común de determinación de las características societarias y laborales de la SE sino fragmentada en una treintena de reglamentos nacionales (Fulton, 2013). La sensación de oportunidad perdida con la SE no es más que un reflejo, por otro lado, del largo impasse que, desde finales de la década de los años noventa, atraviesan las relaciones laborales europeas. La pérdida de dinamismo y la percepción de haber alcanzado techo en procesos tales como el Diálogo Social Europeo interprofesional o los comités de empresa europeos se transmite en este caso a la SE como proyecto de realización no satisfactoria. Las relaciones laborales europeas constituyen un complejo entramado de gobierno multi-nivel que entrelaza a distintos procesos y actores (Leisink y Hyman, 2005). Desde esta perspectiva, aislar el problema del escaso impacto práctico de la SE del momento que atraviesan el resto de espacios de las relaciones laborales europeas no tiene sentido. Unos y otros se ven retroalimentados, tal y como se ha observado al evaluar la contribución de la adopción de la normativa de comités de empresa europeos sobre la aprobación de la regulación de la SE.

No es, sin embargo, pertinente hablar de la SE como una oportunidad perdida para la democracia industrial en Europa puesto que, si bien de forma muy desigual, la norma sienta las bases para la extensión de un modelo multiforme participación de los trabajadores en la toma de decisiones corporativas a escala transnacional. Los problemas no se encuentran tanto en la norma misma como en el espíritu que la guía. La subsidiariedad y el voluntarismo inherente a las formas de regulación de las relaciones laborales europeas de empresa, y su carácter de suaves, negociables o blandas, afecta negativamente a su vigor estandarizador. Lo ha hecho en el caso de los comités de empresa europeos (González Begega, 2011) y lo hace, en mayor medida aún, con la implicación de trabajadores en la SE. En último término, dentro de cada SE se negocia una norma ajustada e individual de derecho a la información, a la consulta y a la participación de los trabajadores (Keller y Werner, 2008; 2012).

En segundo lugar, queda por resolver si los ejemplos de SE con prácticas de participación de empleados en marcha favorecen o amenazan los derechos de los trabajadores en materia de información, consulta y participación y determinar cuál ha sido su impacto sobre la europeización de las relaciones laborales de empresa. Para ello, la evidencia empírica disponible sobre la SE es aún escasa (Rehfeldt et al., 2011). Las experiencias de implicación laboral investigadas muestran una amplia diversidad de comportamientos que apuntan:

a un proceso de aprendizaje a través del cual representantes de trabajadores de diversos orígenes nacionales se encuentran en comités de empresa de la SE y en consejos de administración formando parte de la estructura de gobierno de la empresa pero enfrentados a múltiples incertidumbres en torno al futuro de [su propia participación] (Stollt y Kluge, 2011b: 85).

Algunos retos y dinámicas de la SE recuerdan a los de los comités de empresa europeos. En cualquier caso, los problemas de impacto geográfico desigual, de extrema fragmentación de la normativa sobre la SE y de las implicaciones 
societarias y no solo laborales de este proceso, nos refieren a un escenario cualitativamente distinto.

\section{Bibliografía}

Blackburn, T.L. (1993). The Societas Europaea: the evolving European Corporation Statute. Fordham Law Review. Vol. 61 Núm. 4, 695-773.

Business Europe (2011). Position Paper. Employment involvement in the European Company. Brussels, 6/10/2011.

Carley, M.; Bockler, H. (1998). European Company Statute nearing adoption. European Industrial Relations Review. Vol. 293: 24-27.

Conchon, A. (2011). Board-level employee representation rights in Europe. Facts and trends. Brussels: ETUI.

Conchon, A.; Waddington, J. (2011). Board-level employee representation in Europe: challenging commonplace prejudices. En N. Kluge y S. Vitols, (Eds.), The Sustainable Company: a new approach to corporate governance (pp. 91-111). Brussels: ETUI.

Cremers, J.; Stollt, M. ; Vitols, S. (2013). A decade of experience with the European Company. Brussels: ETUI.

Cremers, J.; Carlson, A. (2013). SEs in the Czech Republic. En J. Cremers, M. Stollt y S. Vitols (Eds.), A decade of experience with the European Company (pp. 107-122). Brussels: ETUI.

Davies, P.L. (2003). Workers on the board of the European company. Industrial Law Journal. Núm. 2, 75-96.

Eidenmüller, H.; Lasak, J. (2011). The Czech Societas Europaea Puzzle. ECGI Law Working Paper. Núm. 183/2011.

Ernst \& Young (2009). Study on the operation and impact of the Statute for a European Company (SE). Report drawn up following call for tender from the European Commission. Ernst \& Young Consulting.

Esteban Velasco, G. (2003). La Sociedad Europea: Un instrumento jurídico al servicio de la reestructuración empresarial. Revista Valenciana de Economía y Hacienda. Vol. 8 Núm. 2, 213-249. 
Esteban Velasco, G. (2007). La implicación de los trabajadores en la sociedad anónima europea en la Ley 31/2006 de transposición de la Directiva europea. Revista de Derecho Bancario y Bursátil. Vol. 26 Núm. 105, 180-193.

Esteban Velasco, G. y Fernández del Pozo, L. (2004). La Sociedad Anónima Europea. Régimen jurídico, societario, laboral y fiscal. Madrid: Marcial Pons.

European Commission (1970). Proposal for a Council Regulation Embodying a Statute for the European Company. COM (70) 600 final. OJC 124, 10/10/1970.

European Commission (1975). Proposal for a Council Regulation on the Statute for European Companies. Amended proposal presented by the Commission to the Council on 13/05/1975, pursuant to the second paragraph of Article 149 of the EEC Treaty. COM (75) 150 final. Bulletin of the EC Supplement 4/75.

European Commission (1988). Statute for the European Company. Internal Market White Paper, point 137. Memorandum from the Commission to Parliament, the Council and the two sides of industry. COM (88) 320 final, 8/06/1988. Bulletin of the EC Supplement 3/88.

European Commission (1989). Statute for a European Company. Proposal for a Regulation on the Statute for a European Company. Proposal for a Directive complementing the Statute for a European Company with regard to the involvement of employees in the European Company. COM (89) 268 final, 25/08/1989. Bulletin of the EC Supplement 5/89.

European Commission (1991a). Amended proposal for a Council Regulation on the Statute for a European Company. COM (91) 174 final, OJC 176, 08/07/1991.

European Commission (1991b). Amended proposal for a Council Directive complementing the Statute for a European company with regard to the involvement of employees in the European company. COM (91) 174 final, 29/05/1991, OJC138/8.

European Commission (2011). Consultation document. First phase consultation of Social Partners under Article 154 TFEU on the possible review of Directive 2001/86/EC supplementing the Statute for a European company with regard to the involvement of employees, Brussels, 5/07/2011, COM (2011) 4707 final.

European Commission (2012). Communication. Action Plan: European company law and corporate governance - a modern legal framework for more engaged shareholders and sustainable companies, Brussels, 12/12/2012, COM (2012) 0740 final. 
ETUC (2011). ETUC response to European Commission's first phase consultation of social partners under Article 154 TFEU on the possible review of Directive 2001/86/EC supplementing the Statute for a European company with regard to the involvement of employees. Brussels, 28/10/2011.

ETUI (2010). ETUI's reply to the consultation of the EU Commission on the SE Study of Ernst \& Young. Brussels: ETUI.

Fulton, L. (2013). From a European blueprint to national law. En J. Cremers, M. Stollt y N. Kluge, (Eds.), A decade of experience with the European Company (pp. 25-48). Brussels: ETUI.

Gold, M.; Nikopoulos, A.; Kluge, N. (2009). The European Company Statute. A new approach to corporate governance. Bern: Peter Lang.

Gold, M.; Schwimbersky, S. (2008). The European Company Statute: implications for industrial relations in the European Union. European Journal of Industrial Relations. Vol. 14 Núm. 1, 44-64.

González Begega, S. (2011). Empresa transnacional y nuevas relaciones laborales. La experiencia de los comités de empresa europeos. Madrid: La Catarata.

González Begega, S.; Köhler, H.D. (2012). La representación de intereses laborales en la Sociedad Anónima Europea (SE). Dimensiones y contenidos. Revista de Derecho Social. Núm. 60, 221-232.

High Level Group on Workers' Involvement (1997). Workers involvement: Davignon Group Final Report, IP/97/396. Brussels, 13/05/1997.

Keller, B.K. (2002). The European Company statute: employee involvement - and beyond. Industrial Relations Journal. Vol. 33 Núm. 5, 424-445.

Keller, B.K.; Werner, F. (2008). The establishment of the European Company: The first cases from an industrial relations perspective. European Journal of Industrial Relations. Vol. 14 Núm. 2, 153-175.

Keller, B.K.; Werner, F. (2012). New forms of employee involvement at European level. The case of the European Company (SE). British Journal of Industrial Relations. Vol. 50 Núm. 4, 620-643.

Kluge, N.; Stollt, M. (2007). La société européenne (SE). Premières experiences de l'application de ce nouvel element du droit européen des sociétés. Examen du point de vue des salaries. En N. Lenoir, (Ed.), La SE ou Societas Europaea. 
Pour une citoyenneté européenne de l'entreprise (pp. 258-267). Paris: La Documentation française.

Knudsen, H.; Müller, H.; Rehfeldt, U. (2008). Converting MAN B\&W Diesel AG into MAN Diesel SE. Negotiations and the agreement on employee involvement”. SEEurope Network Report. Brussels: ETUI.

Köhler, H.D. (2013). La participación de los trabajadores en las empresas alemanas”, Temas para el debate, Vol. 222, 33-35.

Köstler, R. (2013). SEs in Germany. En M. Stollt y S. Vitols, (Eds.), A decade of experience with the European Company (pp. 123-132). Brussels: ETUI.

Köstler, R.; Büggel, A. (2003). The European Company and company law and existing legislative provision for employee participation in the EU Member States. Brussels: ETUI.

Leca, C. (2007). The participation of employees' representatives in the governance structures of the Societas Europaea. European Business Law Review. Vol. 18 Núm. 3, 403-441.

Leisink, P.; Hyman, R. (2005). Introduction: The dual evolution of Europeaisation and varieties of governance. European Journal of Industrial Relations. Vol. 11 Núm. 3, 277-286.

Lenoir, N. (2007). La SE ou Societas Europaea. Pour une citoyenneté européenne de l'entreprise. Paris: La Documentation Française.

Lenoir, N. (2008). The Societas Europaea (SE) in Europe. A promising start and an option with good prospects. Utrecht Law Review. Vol. 4 Núm. 1, 13-21.

Linmondin, K. (2013). The European Company (Societas Europaea). A successful harmonisation of corporate governance in the European Union? Bond Law Review. Vol. 15 Núm. 1, 147-180.

Marshall, T.H.; Bottomore, T. (1950) [1989]. Ciudadanía y clase social. Madrid: Alianza.

Menjucq, M.; Fages F.; Vuiard, L (2008). The European Company under French Law. Main features. European Business Organization Law Review. Núm. 9, 137-154.

McGlynn, C. (1995). European works councils: towards industrial democracy? European Law Journal. Vol. 24 Núm. 1, 78-84. 
Nagels, K.; Sorge, A. (1977). Industrielle Demokratie in Europa. Frankfurt am Main: Campus Verlag.

Rehfeldt, U.; Voss, E.; Pulignano, V.; Kelemen, M.; Telljohann, V.; Fulton, L.; Neuman, L.; Mester, D.; Schütze, K.; Wilke, P. (2011). Employee involvement in companies under the European Company Statute. Dublin: European Foundation for the Improvement of Living and Working Conditions.

Schwinbersky, S.; Gold, M. (2013). The European Company Statute: a tangled history. En J. Cremers, M. Stollt y S. Vitols, (Eds.), A decade of experience with the European Company (pp. 49-66). Brussels: ETUI.

Stollt, M.; Kluge, N. (2011a). La participation des salaries dans la société européenne: vers une européanisation des relations professionelles? La Revue de l'IRES. Vol. 71 Núm. 4, 71-88.

Stollt, M.; Kluge, N. (2011b). The potential of employee involvement in the SE to foster the Europeanization of labour relations. Transfer. European Review of Labour and Research. Vol. 17 Núm. 2, 181-191.

Streeck, W. (1997). Industrial citizenship under regime competition: the case of European works councils. Journal of European Public Policy. Vol. 4 Núm. 4, 643-644.

Taylor, R. (2006). Taking responsibility in a SE. A new challenge for workers from different cultural and political backgrounds”, en N. Kluge y M. Stollt, (Eds.), The European Company. Prospects for worker board-level participation in the Enlarged EU (pp. 93-110). Brussels: ETUI.

Telo, M.; Gobin, C. (1994). Quelle Union Social Européenne? Acquis institutional, acteurs et defis. Bruxelles: Institut d’Etudes Européennes.

Valdés Dal-Ré, F. (2007). Studies on the implementation of labour law directives in the enlarged European Union. Directive 2001/86/EC supplementing the European Company with regard to the involvement of employees. Synthesis report. Madrid: Labour Asociados.

Veersma, U.; Swinkels, S. (2005). Participation in European Companies: views from social partners in three Member States. Transfer: European Review of Labour and Research. Núm. 11 Vol. 2, 189-205.

Wenz, M. (2004). Societas Europaea: Flagship for Company Law - Catalyst for EC Tax Law. European Taxation.Vol. 44, 1-45. 